\title{
Homocysteine induces blood vessel global hypomethylation mediated by LOX-1
}

\author{
X.L. Yang ${ }^{1,2}$, J. Tian ${ }^{1,2}$, Y. Liang ${ }^{2}$, C.J. Ma ${ }^{3}$, A.N. Yang' ${ }^{1}$, J. Wang ${ }^{2}$, \\ S.C. $\mathrm{Ma}^{2}$, Y. Cheng ${ }^{2,3}, \mathrm{X}$. Hua ${ }^{1}$ and Y.D. Jiang ${ }^{1,2}$ \\ ${ }^{1}$ Department of Pathophysiology, Basic Medical School, \\ Ningxia Medical University, Yinchuan, Ningxia, China \\ ${ }^{2}$ Key Laboratory of Cardio-Cerebro-Vascular Diseases, \\ Ningxia Medical University, Yinchuan, Ningxia, China \\ ${ }^{3}$ Department of Clinical Examination, Ningxia Medical University, \\ Yinchuan, Ningxia, China \\ Corresponding author: Y.D. Jiang \\ E-mail: yangwj04@126.com
}

Genet. Mol. Res. 13 (2): 3787-3799 (2014)

Received December 7, 2012

Accepted July 19, 2013

Published May 16, 2014

DOI http://dx.doi.org/10.4238/2014.May.16.2

\begin{abstract}
Homocysteine (Hcy) is an independent risk factor of atherosclerosis through its involvement with the methionine cycle. In this study, we aimed to determine the blood vessel global methylation rate in Hcy-induced atherosclerosis in apolipoprotein-E-deficient $\left(A p o E^{-/-}\right)$mice, and to explore the possible mechanism of this change in endothelial cells. ApoE ${ }^{-/}$mice were divided into a hyperlipidemia (HLP) group, a hyperhomocysteinemia (HHcy) group, and an HHcy + folate + vitamin B12 (HHcy+FA+VB) group. Wild-type C57BL/6J mice were prepared as controls. Total Hcy, lipids, S-adenosylmethionine (SAM), and S-adenosylhomocysteine (SAH) contents in serum were measured with an automatic biochemistry analyzer and high-performance liquid chromatography. Methylation of B1 repetitive elements in blood vessels was tested using nested methylation-specific-polymerase chain reaction (nMS-PCR). Endothelial cells (ECs) were pretreated with Hcy or by adding FA and VB. Lectin-like oxidized LDL receptor-1 (LOX-1) expressions
\end{abstract}


were determined by quantitative PCR, Western blot, and nMS-PCR. The HHcy group displayed severe HLP and HHcy. SAM and SAH contents were also elevated in the HHcy group compared with other groups. Methylation of B1 repetitive elements was significantly increased in the HHcy group $(0.5050 \pm 0.0182)$ compared to the HLP $(0.5158 \pm 0.0163)$ and control $(0.5589 \pm 0.0236)$ groups. mRNA and protein expressions of LOX-1 increased $(0.2877 \pm 0.0341,0.6090 \pm 0.0547)$, whereas methylation expression decreased $(0.5527 \pm 0.0148)$ after $100 \mu \mathrm{M}$ Hcy stimulation in ECs. In conclusion, Hcy-induced atherosclerosis was closely associated with induced hypomethylation status in the blood vessel, and this process was partially mediated by LOX-1 DNA methylation.

Key words: Homocysteine; Global DNA methylation; Atherosclerosis; Lectin-like oxidized LDL receptor-1

\section{INTRODUCTION}

Atherosclerosis is a common pathophysiological process of multiple cardiovascular diseases. Lipid deposition, oxidative stress, immune inflammatory injury, macrophage receptor deficiency, and smooth muscle cell activation are all involved in the development of atherosclerosis (Rapava et al., 2006). As an important independent risk factor of atherosclerosis, homocysteine (Hcy) can influence the atherosclerosis process in various aspects, such as through transmethylation, endothelial injury, oxidative stress, and inflammation response (Tehlivets, 2011).

Hcy is a sulfur-containing amino acid and is an important intermediate metabolism product in the folic acid and methionine cycle. Hcy is derived from methionine, which is used for synthesis of S-adenosylmethionine (SAM) and conversion of Hcy and S-adenosylhomocysteine (SAH) (King et al., 2012). SAM is the only one-carbon unit methyl donor for more than 100 different transmethylation reactions, including DNA and RNA methylation (Kirsch et al., 2009). DNA methylation is a form of epigenetic gene regulation that, combined with the altered binding profile of transcription factors, commonly leads to suppression of gene expression when occurring in a regulatory region (Du et al., 2012). Hypermethylation of $\mathrm{CpG}$ islands in promoter areas results in decreased gene expression, whereas promoters of actively transcribed genes remain nonmethylated (Du et al., 2012). Alterations of DNA methylation patterns have been associated with various diseases, including cancers and cardiovascular diseases (Wernimont et al., 2011; DelgadoCruzata et al., 2012). Lower global DNA methylation has been observed in individuals with severe metabolic syndrome (Turcot et al., 2012). Patients with vascular disease exhibit increased plasma total Hcy (tHcy) and decreased genomic DNA methylation (Castro et al., 2003). Therefore, we speculated that there might be a distinct methylation status in arteries depending on the presence or absence of Hcy. Consistent with our assumption, we found a statistically significant difference in global DNA methylation, as characterized in B1 repetitive elements in the blood vessels of mice, after Hcy stimulation. However, to date, the mechanism involved in the effect of Hcy on the blood vessel methylation status is not very clear.

Condensation of Hcy and serine can form cystathionine in a reaction catalyzed by cystathionine beta-synthase (MacLean et al., 2012), which is another major mechanism in the Hcy metabolic pathway. Hcy contains a free sulfhydryl group, and oxidizes with other thiols to form 
mixed disulfides, which results in the oxidization of other substances, including low-density lipoprotein (LDL). Oxidized modified LDL (Ox-LDL) is generated from LDL oxidative modification, and is an important risk factor of atherosclerosis. The lectin-like oxidized LDL receptor-1 (LOX-1), one of the surface scavenger receptors for Ox-LDL, might mediate the binding and internalization of Ox-LDL to cells, and might be a target regulator that is involved in atherosclerosis (Yoshimoto et al., 2011). Activation of LOX-1 with Ox-LDL induces cell injury in human coronary artery endothelial cells (ECs) (Morawietz, 2010). Expression of LOX-1 in atherosclerotic lesions was increased in mice fed a hypermethionine diet (Thampi et al., 2008). However, to our knowledge, precisely how Hcy affects LOX-1 expression in ECs remains unknown.

In this study, we investigated global DNA methylation in blood vessels in hyperhomocysteinemia (HHcy), and analyzed LOX-1 methylation in cultured ECs in the presence of Hcy. We aimed to find a key factor for further elucidating the mechanism underlying Hcy-induced atherosclerosis.

\section{MATERIAL AND METHODS}

\section{Animals and diets}

Animal procedures were performed in accordance with institutional guidelines under the China animal protection law. Six-week-old male apolipoprotein-E-deficient $\left(\mathrm{ApoE}^{-/}\right)$mice (C57BL/6J genetic background) and C57BL/6J mice were obtained from the Animal Center of Peking University (Beijing, China). Animals were housed individually per cage, and maintained under standard environmental conditions (12-h light/dark cycle at $22^{\circ}-24^{\circ} \mathrm{C}$ and $40-60 \%$ humidity). After 1 week of acclimation, the mice were divided into four groups and maintained for 15 weeks on the following diets: 1 ) the control group (wild-type $\mathrm{C} 57 \mathrm{BL} / 6 \mathrm{~J}$ mice) was fed regular mouse diet ( $0 \%$ cholesterol, $5.23 \%$ fat, $0.37 \%$ methionine, $2.39 \mathrm{mg} / \mathrm{g}$ choline, $3.19 \mathrm{mg} / \mathrm{kg}$ folate (FA), 54.6 $\mu \mathrm{g} / \mathrm{kg}$ vitamin B12 (VB), $14.5 \mathrm{mg} / \mathrm{kg}$ vitamin B6; catalog No. 8640, Harlan Teklad, Madison, WI, USA), 2) the hyperlipidemia (HLP) group (ApoE ${ }^{-1}$ mice) was fed regular mouse diet, 3) the HHcy group (ApoE ${ }^{-/}$mice) was fed a high-fat diet with $1.7 \%$ methionine supplement, and 4) the HHcy $+\mathrm{FA}+\mathrm{VB}$ group (ApoE $\mathrm{E}^{-/}$mice) was fed a high-fat diet plus $1.7 \%$ methionine, FA, and VB. The animals were managed using welfare animal practices.

\section{EC culture}

The EC line was obtained from Si Chuan University (China). ECs were adjusted to a concentration of $5 \times 10^{5} / \mathrm{mL}$ in complete RPMI-1640 medium containing $10 \%$ heat-inactivated fetal bovine serum, $100 \mathrm{mg} / \mathrm{mL}$ streptomycin, and $100 \mathrm{U} / \mathrm{mL}$ penicillin. Then, different Hcy concentrations $(0,50,100,200$, and $500 \mu \mathrm{M}$; Sigma, USA) were added, and cells were incubated at $37^{\circ} \mathrm{C}$ in a $5 \% \mathrm{CO}_{2}$ humidified incubator. After a 72 -h incubation, cells and supernatants were collected for further use.

\section{Determination of serum Hcy, SAM, SAH, and lipid concentrations}

After 15 weeks of feeding, mice were fasted for $4 \mathrm{~h}$, anesthetized with intra-peritoneal pentobarbital (60 mg/kg Nembutal), and blood was obtained by intra-cardiac puncture. Serum was 
isolated by centrifugation at $1000 \mathrm{~g}$ for $10 \mathrm{~min}$ at $4{ }^{\circ} \mathrm{C}$ and stored at $-80^{\circ} \mathrm{C}$. tHcy and lipid [total cholesterol (TC), triglyceride (TG), and LDL] concentrations in serum were measured with the automatic ADVIA 2400 Automatic Biochemistry Analyzer (Siemens, Munich, Germany). Serum SAM and SAH concentrations were detected using high-performance liquid chromatography (HPLC).

\section{Nested methylation-specific-polymerase chain reaction (nMS-PCR)}

Global DNA was isolated from arteries and ECs using the Wizard ${ }^{\circledR}$ Genomic DNA Purification Kit (Promega, USA) following manufacturer protocols. DNA concentration was assessed with an ND-1000 spectrophotometer (NanoDrop, Wilmington, DE, USA). DNA denaturation and bisulfite conversion processes were integrated into one step using the EZ DNA Methylation-Gold ${ }^{\mathrm{TM}}$ Kit (ZYMO, Los Angeles, CA, USA) according to manufacturer instructions. Methylation patterns of the B1 repetitive elements and LOX-1 were confirmed with nMS-PCR analysis, which consists of two-step PCR amplifications. Methylation patterns of the B1 repetitive elements and LOX-1 were confirmed with nMS-PCR analysis. A three-temperature touchdown PCR program was used for DNA amplification: $94^{\circ} \mathrm{C}$ for $5 \mathrm{~min} ; 30$ cycles at $94^{\circ} \mathrm{C}$ for $30 \mathrm{~s}$, annealing temperature for $30 \mathrm{~s}$ (decreasing $1^{\circ}$ or $0.5^{\circ} \mathrm{C}$ between two cycles), $72^{\circ} \mathrm{C}$ for $1 \mathrm{~min}$; an additional 20 cycles at $94^{\circ} \mathrm{C}$ for $30 \mathrm{~s}$, annealing temperature for $30 \mathrm{~s}, 72^{\circ} \mathrm{C}$ for $1 \mathrm{~min}$; and a final extension at $72^{\circ} \mathrm{C}$ for $7 \mathrm{~min}$ (Table 1). The amplification products were analyzed by agarose gel electrophoresis.

\begin{tabular}{|c|c|c|c|c|}
\hline Primers & Sequence $\left(5^{\prime}-3^{\prime}\right)$ & $\mathrm{Ta}\left({ }^{\circ} \mathrm{C}\right)$ & $\operatorname{Tm}\left({ }^{\circ} \mathrm{C}\right)$ & Length (bp) \\
\hline B1-O & $\begin{array}{l}\text { Sense: ATAGAAGTGGATGTTTATAGTTAGTTATTG } \\
\text { Antisense: CACTCCAACTTTTTAACCCTAAC }\end{array}$ & 56.0 & 41.0 & 269 \\
\hline B1-M & $\begin{array}{l}\text { Sense: GTTAGTTATTGGATGGGTTATACGG } \\
\text { Antisense: TACAACTAAAAACAAAAACTCCGAA }\end{array}$ & 63.7 & 48.7 & 139 \\
\hline B1-U & $\begin{array}{l}\text { Sense: GTTAGTTATTGGATGGGTTATATGG } \\
\text { Antisense: TACAACTAAAAACAAAAACTCCAAA }\end{array}$ & 63.7 & 48.7 & 139 \\
\hline LOX-1-O & $\begin{array}{l}\text { Sense: TTAGTATTGTGGGAGGTTGAGGTAG } \\
\text { Antisense: TAAAATTTCACCCTTATTACCCAAA }\end{array}$ & 68.3 & 53.3 & 251 \\
\hline LOX-1-M & $\begin{array}{l}\text { Sense: TTGAAAATATAAAATAATTAGTCGG } \\
\text { Antisense: TAAATTACAATAACATAATCTCG }\end{array}$ & 66.0 & 51.0 & 137 \\
\hline LOX-1-U & $\begin{array}{l}\text { Sense: TTGAAAATATAAAATAATTAGTTGG } \\
\text { Antisense: AATAAATTACAATAACATAATCTCAAC }\end{array}$ & 66.0 & 51.0 & 139 \\
\hline
\end{tabular}

$\mathrm{B} 1=\mathrm{B} 1$ repetitive elements; $\mathrm{O}=$ outer primers; $\mathrm{M}=$ methylated primers; $\mathrm{U}=$ unmethylated primers; $\mathrm{Ta}=$ annealing temperature; $\mathrm{Tm}=$ melting temperature.

\section{Fluorescence reverse transcriptase-quantitative PCR (RT-qPCR)}

The relative expression of DNA methyltransferase 1 (DNMT1) mRNA was examined using a quantitative PCR System (Bio-Rad, Hercules, CA, USA). Briefly, total RNA was isolated from ECs in $1 \mathrm{~mL}$ Trizol (Invitrogen, Carlsbad, CA, USA). The concentration of isolated RNA was determined with a spectrophotometer. Then, total RNA was reverse transcribed using the Revertid ${ }^{\mathrm{TM}}$ First-Strand cDNA Synthesis Kit (Thermo Scientific, Glen Burnie, MD, USA) according to manufacturer instructions. RT-qPCR was performed with the SYBR Green real-time PCR master mix kit (MBI). Two-microgram cDNA templates were applied in all reactions. Reactions with each primer pair and template were performed in triplicate. An amplification curve was obtained for each PCR run. The relative change in DNMT1 mRNA 
expression was determined by fold-change analysis, in which the degree of change $=2^{-\Delta \Delta C t}$, where cycle threshold $(\mathrm{Ct})=\left(\mathrm{Ct}_{\mathrm{DNMT} 1}-\mathrm{Ct}_{\mathrm{GAPDH}}\right)$ treatment $-\left(\mathrm{Ct}_{\mathrm{DNMT1}}-\mathrm{Ct}_{\mathrm{GAPDH}}\right)$ control. The relative expression of LOX-1 mRNA in ECs was determined in the same manner.

The primers used were as follows: DNMT1 sense (S): 5'-GGAGCCCAGCAAGAG TA-3', DNMT1 antisense (A): 5'-GGGAGACACCAGCCAAAT-3', LOX-1 S: 5'-AATGATA GAAACCCTTGC-3', LOX-1 A: 5'-TTCCCAGTTAAATGAGCC-3', GAPDH S: 5'-AGAAGGCT GGGGCTCATTTG-3', GAPDH A: 5'-AGGGGCCACAGTCTTC-3'.

\section{Western blot analysis}

ECs were lysed in RIPA buffer (Pierce, Rockford, IL, USA) supplemented with $1 \mathrm{mM}$ protease inhibitors (Roche, USA). After quantification (BCA protein assay, Pierce), proteins were separated by sodium dodecyl sulfate-polyacrylamide gel electrophoresis (SDS-PAGE) and transferred to polyvinylidene difluoride membranes. The membranes were then blocked with $5 \%$ skim milk for $1 \mathrm{~h}$ and incubated overnight at $4^{\circ} \mathrm{C}$ with anti- $\beta$-actin $(1: 1000$, Sigma, St. Louis, MO, USA) or anti-LOX-1 (1:1000, Santa Cruz Biotechnology, USA). Subsequently, the membranes were incubated with appropriate secondary antibodies (Amersham Biosciences, Piscataway, NJ, USA). After washing, proteins were detected using an enhanced chemiluminescence system (ECL Plus, Amersham Biosciences, Uppsala, Sweden).

\section{Enzyme-linked immunosorbent assay (ELISA)}

An ELISA for Ox-LDL in ECs was performed using Ox-LDL ELISA kits (Adliteram Diagnostic Laboratories Inc., USA) according to manufacturer instructions. The concentrations of Ox-LDL were quantified in biomedical units as defined by the manufacturer. The concentration of the Ox-LDL was determined by establishing a standard curve.

\section{Statistical analysis}

For comparisons between two groups, the two-sided unpaired Student $t$-test was used with $\mathrm{P}<0.05$ being considered significant. For multiple group comparisons, one-way analysis of variance (ANOVA) was carried out followed by Tukey multiple comparison tests using the GraphPad Prism 5.0 software.

\section{RESULTS}

\section{ApoE $^{-/-}$mice have HHcy and HLP}

HHcy and HLP are usually present simultaneously. In order to explore whether the methionine diet induced HHcy and HLP in $\mathrm{ApoE}^{-/}$mice, we measured serum tHcy and lipid levels in mice. After 15 weeks on experimental diets, compared to the control group, serum tHcy levels were significantly elevated by approximately 1.2-, 2.5-, and 1.7-fold in the HLP, HHcy, and HHcy+FA+VB groups, respectively $(\mathrm{P}<0.05$; Figure 1$)$. The HHcy group showed the highest tHcy level among all groups $(\mathrm{P}<0.05)$. Serum tHcy levels decreased by $23 \%$ in the HHcy+FA+VB group compared to the HHcy group $(\mathrm{P}<0.05)$. Consistent with this obser- 
vation, we found that serum lipid levels, including TC, TG, and LDL, were also higher in the HLP group than in the control group $(\mathrm{P}<0.05$; Table 2$)$. Serum TC, TG, and LDL levels increased by 52,72 , and $138 \%$, respectively, in the HHcy group compared with the HLP group. The HHcy+FA+VB group, which was fed with a high-methionine diet plus folate and vitamin $\mathrm{B} 12$ in $\mathrm{ApoE}^{-/}$background mice, displayed lower TC, TG, and LDL levels than the HHcy group $(\mathrm{P}<0.05$; Table 2$)$. These findings indicated that HHcy and HLP were not only present simultaneously in $\mathrm{ApoE}^{-/-}$mice, but also aggravated each other.

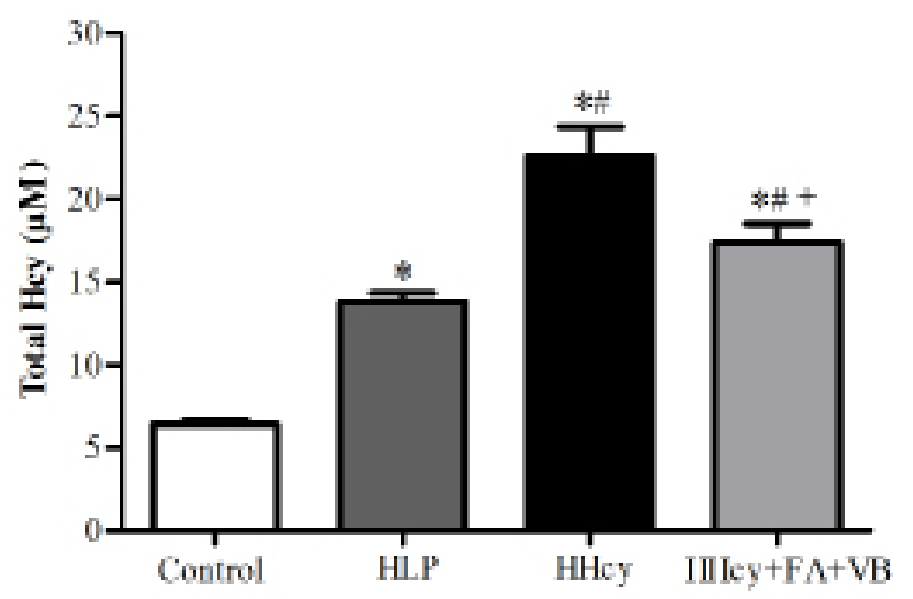

Figure 1. Levels of serum total homocysteine (Hcy) in $\mathrm{ApoE}^{-/-}$mice. $\mathrm{ApoE}^{-/-}$mice were executed and serum total Hcy was detected by the automatic biochemistry analyzer. Results are reported as means $\pm \mathrm{SE}(\mathrm{N}=10)$. $* \mathrm{P}<$ 0.05 vs control; ${ }^{\#} \mathrm{P}<0.05$ vs HLP; ${ }^{+} \mathrm{P}<0.05$ vs HHcy. HLP $=$ hyperlipidemia; HHcy $=$ hyperhomocysteinemia; $\mathrm{HHcy}+\mathrm{FA}+\mathrm{VB}=\mathrm{HHcy}$ supplemented with folate and vitamin B12.

Table 2. Levels of lipids in $\mathrm{ApoE}^{-/-}$mice.

\begin{tabular}{lcccc}
\hline Group & TC $(\mathrm{mM})$ & TG $(\mathrm{mM})$ & LDL $(\mu \mathrm{M})$ & Ox-LDL \\
\hline Control & $2.06 \pm 0.11$ & $0.43 \pm 0.14$ & $0.41 \pm 0.01$ & $52.60 \pm 3.93$ \\
HLP & $15.83 \pm 1.06^{*}$ & $0.92 \pm 0.16^{*}$ & $0.79 \pm 0.07^{*}$ & $67.33 \pm 2.92$ \\
HHcy & $24.02 \pm 2.20^{\#}$ & $1.58 \pm 0.22^{*}$ & $1.88 \pm 0.42^{\#}$ & $106.80 \pm 10.56^{\#}$ \\
HHcy+FA+VB & $11.32 \pm 0.83^{+}$ & $0.77 \pm 0.19^{+}$ & $0.62 \pm 0.05^{+}$ & $70.94 \pm 3.16^{+}$ \\
\hline
\end{tabular}

Data are reported as means $\pm \mathrm{SE}$ for $\mathrm{N}=10$. After 15 weeks of feeding, blood was collected and serum lipids were detected by an automatic biochemistry analyzer. ${ }^{*} \mathrm{P}<0.05$ vs control; ${ }^{*} \mathrm{P}<0.05$ vs HLP; ${ }^{+} \mathrm{P}<0.05$ vs HHcy. $\mathrm{TC}=$ total cholesterol; $\mathrm{TG}=$ triglyceride; $\mathrm{LDL}=$ low-density lipoprotein; Ox-LDL $=$ oxidized LDL. For other abbreviations, see legend to Figure 1.

\section{Hcy induced high concentration of serum SAM}

SAM is an important methyl donor in vivo, which is converted to SAH by the removal of the S-methyl group during the transmethylation reaction. After the mice were fed different diets for 15 weeks, serum concentrations of SAM increased by approximately 3.0-, 3.6-, and 2.5-fold in the HLP, HHcy, and HHcy+FA+VB groups, respectively, compared to the control group $(\mathrm{P}<0.01$, Figure $2 \mathrm{~A})$. The HHcy group displayed a higher SAM concentration than the HLP group $(\mathrm{P}<0.05)$. After treatment with folate and vitamin $\mathrm{B} 12$, we observed a $25 \%$ 
decreased concentration of SAM in serum compared with the HHcy group $(\mathrm{P}<0.05)$. A similar trend was observed for the serum SAH concentration; all $\mathrm{ApoE}^{-/-}$groups displayed higher concentrations of SAH than the control group $(\mathrm{P}<0.05$; Figure $2 \mathrm{~B})$. These findings indicated that methylated activity was increased after Hcy stimulation.

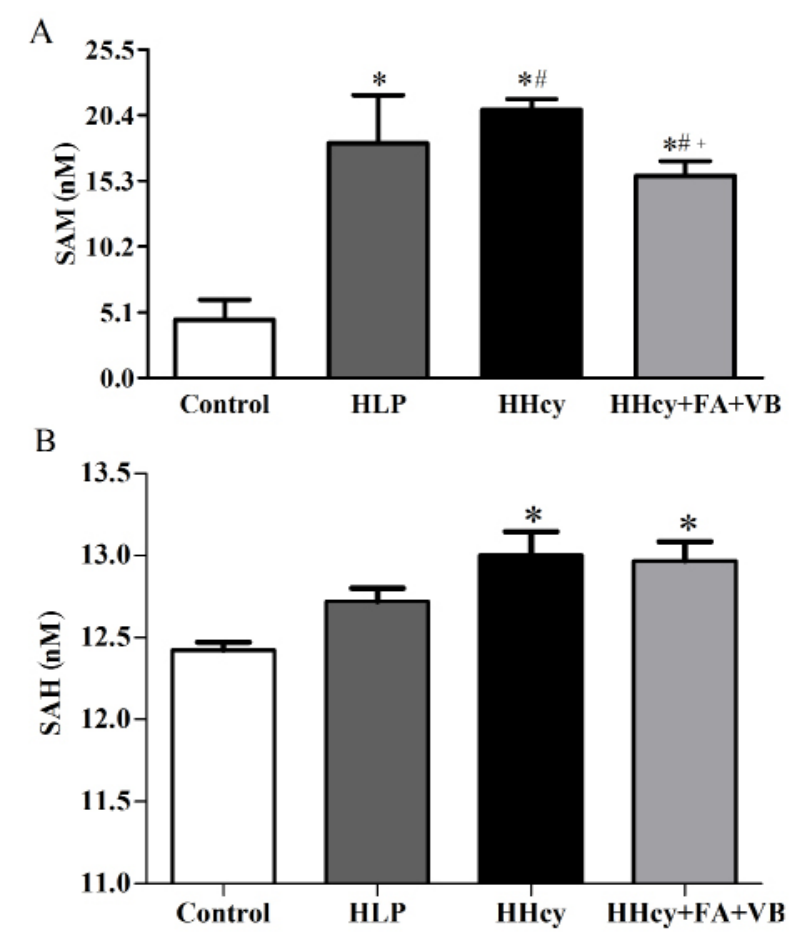

Figure 2. Results are reported as means $\pm \mathrm{SE}(\mathrm{N}=10)$. Serum SAM and $\mathrm{SAH}$ concentrations in $\mathrm{ApoE}^{-/-}$mice fed different diets. After 15 weeks, blood was collected and serum SAM was detected by HPLC. $* \mathrm{P}<0.05$ vs control; ${ }^{*} \mathrm{P}<0.05$ vs HLP; ${ }^{+} \mathrm{P}<0.05$ vs HHcy. HLP = hyperlipidemia; HHcy = hyperhomocysteinemia; HHcy+FA+VB = hyperhomocysteinemia supplemented with folate and vitamin B12; SAM $=$ S-adenosylmethionine; $\mathrm{SAH}=$ S-adenosylhomocysteine.

\section{Hcy induced B1 repetitive element hypomethylation in blood vessels}

B1 repetitive elements have been preferentially retained in close proximity to genes that perform specific functions in the cell, which are located in a mouse methylation center, and provide a target for de novo DNA methylation. Methylation of B1 repetitive elements can repress its expression. We therefore determined the DNA methylation status of B1 repetitive elements in different tissues using nMS-PCR. DNA methylation of B1 repetitive elements in the HLP and HHcy groups decreased by approximately 7.71 and $9.67 \%$, respectively, compared to the control group $(\mathrm{P}<0.05$; Figure 3). After treatment with FA and VB, we observed a $9.78 \%$ increase in DNA methylation of $\mathrm{B} 1$ repetitive elements in blood vessels $(\mathrm{P}<0.05)$. Although a slight difference in methylation of B1 repetitive elements in blood vessels was observed between the HLP and HHcy groups, the difference was not statistically significant $(\mathrm{P}>0.05)$. Thus, we speculated that global DNA methylation of blood vessels is an important feature in Hcy-induced atherosclerosis. 

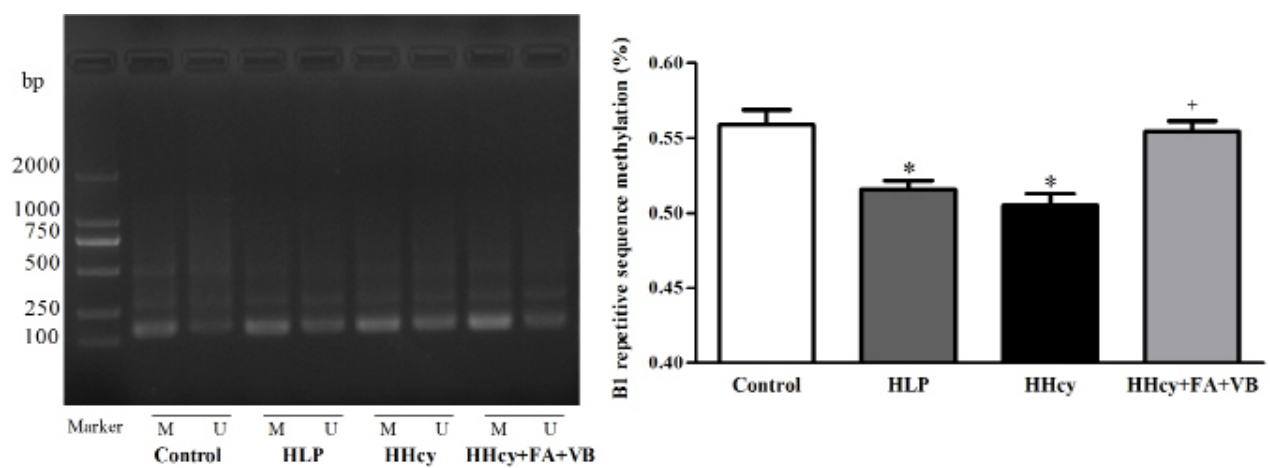

Figure 3. Methylation expression of B1 repetitive elements in mice. After 15 weeks feeding different diets, blood vessels were isolated and DNA was extracted. DNA methylation status of B1 repetitive elements was determined by nested methylation-specific-polymerase chain reaction (nMS-PCR), and relative expression methylation of B1 repetitive elements was calculated. Values are reported as means $\pm \mathrm{SEM}(\mathrm{N}=10) .{ }^{*} \mathrm{P}<0.05$ vs control; ${ }^{+} \mathrm{P}<0.05$ vs $\mathrm{HHcy} . \mathrm{M}=$ methylated PCR products; $\mathrm{U}=$ unmethylated PCR products. For abbreviations, see legend to Figure 1.

\section{Hcy restrained DNMT1 expression}

DNMTs are key enzymes involved in the DNA methylation process with respect to reactions of methyl groups transferred to the C5 position of the cytosine base. DNMT1, the first isolated and purified methyltransferase, is crucial for genomic integrity by preserving DNA methylation patterns throughout development and during whole life periods. Our results showed that the mRNA expressions of DNMT1 in ECs were degrading with increasing Hcy concentrations. Hcy concentrations of 50, 100, 200, and $500 \mu \mathrm{M}$ showed 20, 78.06, 84.0, and $90.37 \%$ decreased expression compared to the control group $(0 \mu \mathrm{M}$ Hcy group; $\mathrm{P}<0.05$ or $\mathrm{P}$ $<0.01$, Figure 4). ECs incubated with $100 \mu \mathrm{M} \mathrm{Hcy}$ and FA and VB exhibited approximately $346.38 \%$ increased expression levels compared to the Hcy group. These data revealed that the expression of DNMT1 might be suppressed by Hcy in ECs.
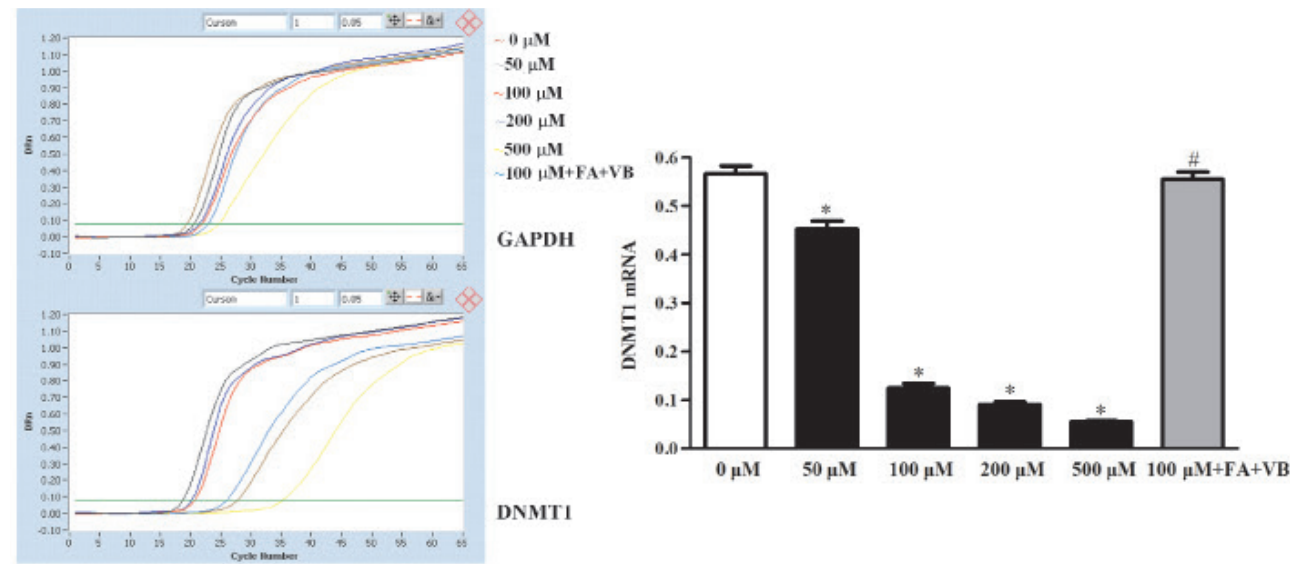

DNMTI

Figure 4. mRNA expression of DNMT1 in endothelial cells. Total RNA was extracted and cDNA was synthetized. Then, mRNA levels of DNMT1 in endothelial cells were determined by qPCR. Data are reported as means $\pm \mathrm{SE}$. ${ }^{*} \mathrm{P}<0.05$ vs $0 \mu \mathrm{M}$ group, ${ }^{\sharp} \mathrm{P}<0.05$ vs $100 \mu \mathrm{M}$ Hcy group. The results were repeated three times. 


\section{Hcy increased LOX-1 mRNA and protein expression but weakened its methylation}

LOX-1 is expressed in ECs, macrophages, and smooth muscle cells, and plays crucial roles in atherosclerotic pathogenesis. In the presence of Hcy, the mRNA expressions of LOX1 were increased by approximately 1.46-, 4.10-, 9.20-, and 15.82-fold under different Hcy concentrations $(50,100,200$, and $500 \mu \mathrm{M})$ compared to the control group $(\mathrm{P}<0.05$; Figure $5 \mathrm{~A})$. After treatment with FA and VB, mRNA expression of LOX-1 was decreased by $60.79 \%$ compared to the $100 \mu \mathrm{M}$ Hcy group. Consistent with the mRNA expression results, protein expression of LOX-1 also increased in the Hcy groups (Figure 5B). Concentrations of 100, 200, and $500 \mu \mathrm{M}$ Hcy elevated LOX-1 by approximately $24.97,34.97$, and $42.21 \%$, respectively, compared to the control group $(\mathrm{P}<0.05$ and $\mathrm{P}<0.01)$. In the FA and VB group, LOX-1 protein expression was degraded by approximately $5.30 \%$ compared to the $100 \mu \mathrm{M}$ Hcy group $(\mathrm{P}>$ 0.05). In general, hypermethylation of DNA promoter regions inhibits gene expression. Therefore, we further detected LOX-1 promoter region methylation by nMS-PCR. As expected, Hcy significantly induced LOX-1 methylation compared to the control group $(\mathrm{P}<0.01$; Figure $5 \mathrm{C})$. Concentrations of 100,200 , and $500 \mu \mathrm{M}$ Hcy degraded LOX-1 by 5.76, 7.62, and 9.62\%, respectively, compared to the control group $(\mathrm{P}<0.05$ and $\mathrm{P}<0.01)$. We also found a $15 \%$ reduction of LOX-1 methylation in the FA and VB group compared with the $100 \mu \mathrm{M}$ Hcy group. These results suggested that Hcy weakened LOX-1 expression both at the transcriptional and translational levels by boosting LOX-1 gene promoter region methylation.

\section{Ox-LDL concentration in ECs}

Ox-LDL has long been considered to be a factor involved in the initiation and progression of atherosclerotic plaques. Similar to the protein expression of LOX-1, the concentrations of Ox-LDL were increased with increasing Hcy concentrations in ECs. Ox-LDL concentration in the $50,100,200$, and $500 \mu \mathrm{M}$ Hcy groups increased by approximately $20.35,43.66,53.56$, and $108.31 \%$ compared to the control group $(0 \mu \mathrm{M} \mathrm{Hcy}, \mathrm{P}<0.05$; Figure 6$)$. In the FA and VB group, there was a $20.55 \%$ decrease in Ox-LDL concentration compared to the $100 \mu \mathrm{M}$ Hcy group $(\mathrm{P}<0.05)$.

\section{DISCUSSION}

In this study, we determined the global methylation of blood vessels in HHcy mice and evaluated LOX-1 expression in HHcy ECs. We found a significant difference in global DNA methylation rates in blood vessels of mice in the presence of HHcy. Moreover, LOX-1 DNA showed hypomethylation in HHcy ECs.

Serum lipoprotein ApoE can clear Hcy, which accelerates the generation and development of atherosclerosis. Deficiencies in the ApoE gene or knockout ApoE genes may cause HHcy. We found that serum tHcy levels in the HLA group (ApoE ${ }^{-/}$mice) were higher than in controls, which indicated that the construction of the HHcy model was successful. We also confirmed that serum tHcy and lipid levels, including TC, TG, LDL, and Ox-LDL, were highest in the HHcy group among all groups. These results suggested that HLP and HHcy might aggravate each other, the effect of HLA and HHcy were not independent, and there might be a mechanism connecting HLP with HHcy. 


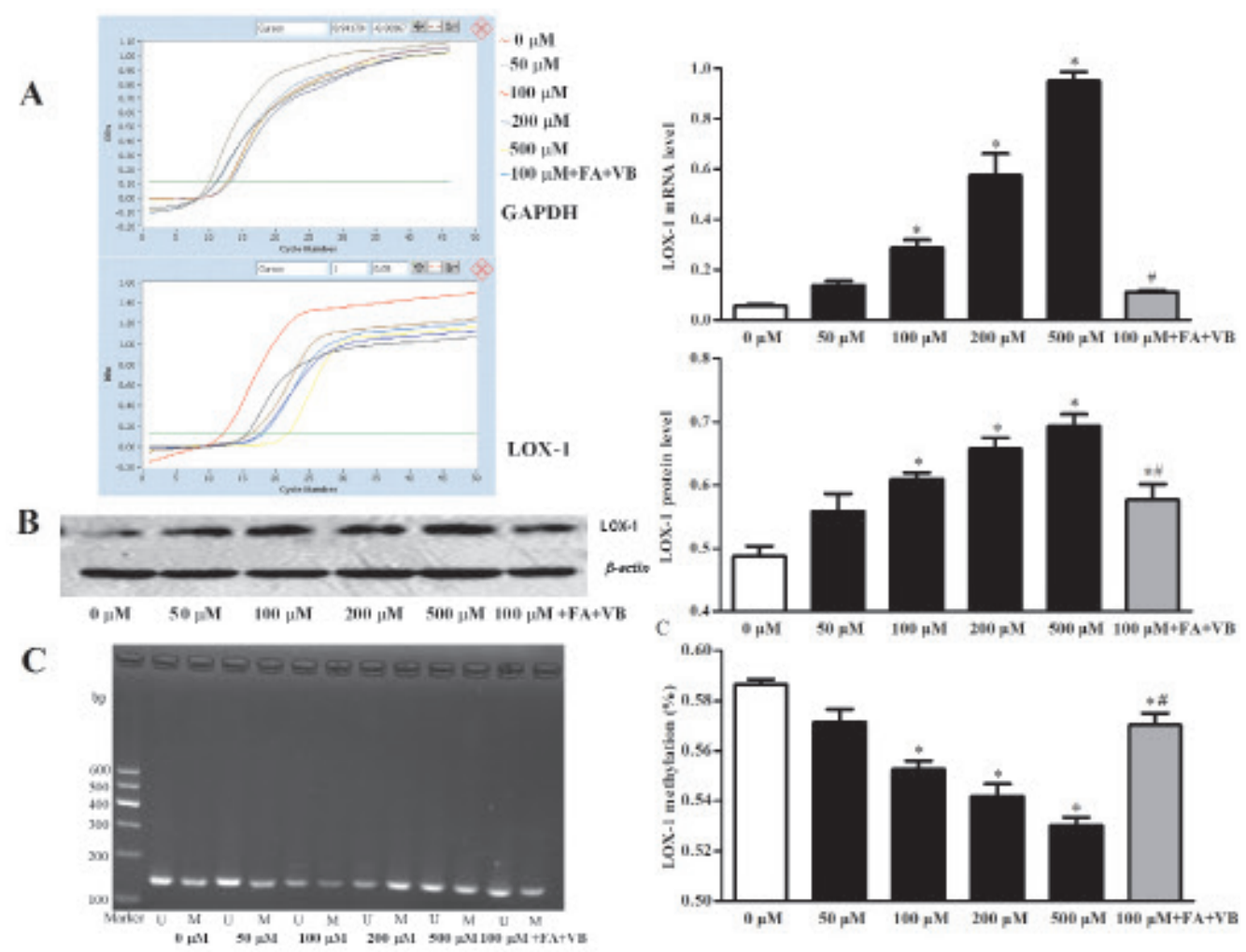

Figure 5. The mRNA, protein and methylation expressions of LOX-1 in endothelial cells. After Hcy stimulation, ECs were collected. Total RNA, protein and DNA were extracted for detecting the expression of mRNA (A), protein $(\mathbf{B})$ and methylation $(\mathbf{C})$ by qRT-PCR, Western blot and nMS-PCR. Data are reported as means \pm SE. $* \mathrm{P}<$ 0.05 vs $0 \mu \mathrm{M}$ Hcy group, ${ }^{\#} \mathrm{P}<0.05$ vs $100 \mu \mathrm{M}$ Hcy group. The results were repeated three times. $\mathrm{M}=$ methylated PCR products; $\mathrm{U}=$ unmethylated PCR products.

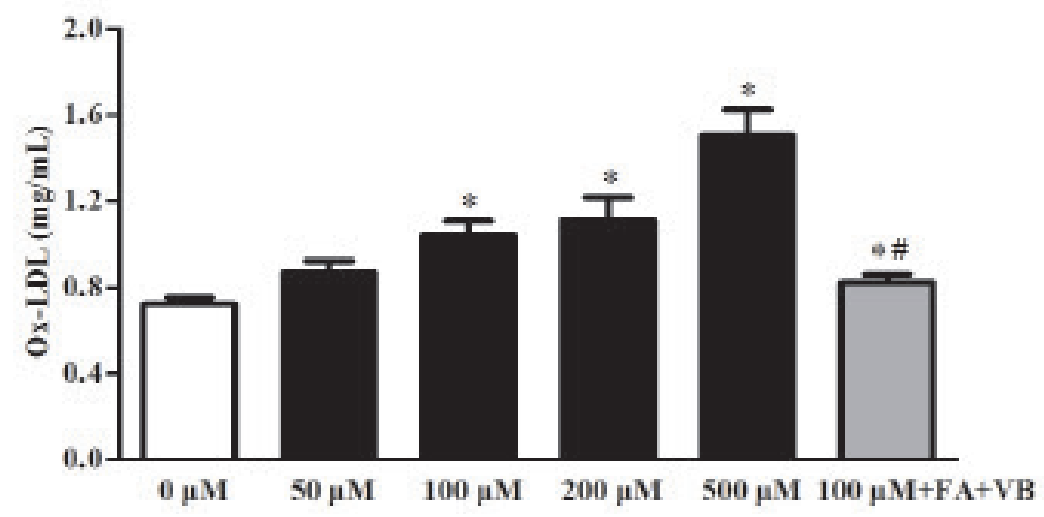

Figure 6. Ox-LDL concentrations in endothelial cells. Enzyme-linked immunosorbent assay (ELISA) was carried out for detection of Ox-LDL in ECs. Data are reported as means $\pm \mathrm{SE}$. ${ }^{*} \mathrm{P}<0.05$ vs $0 \mu \mathrm{M}$ Hcy group, ${ }^{*} \mathrm{P}<0.05$ vs $100 \mu \mathrm{M}$ Hcy group. The results were repeated three times. 
One-carbon metabolism is critical for the availability of methyl groups and therefore for the methylation of DNA, which might affect both hypomethylation and hypermethylation. $\mathrm{SAH}$, which is formed after donation of the methyl group of SAM to a methyl acceptor, is reversibly hydrolyzed to adenosine and Hcy by S-adenosylhomocysteine hydrolase. Increased SAM concentration might represent an active methylation status. HHcy induced higher concentrations of SAM and SAH than HLA, which indicated that transmethylation was augmented in the presence of Hcy. DNMT catalyzes the transfer of a methyl group from SAM to the $\mathrm{C} 5$ of cytosine within $\mathrm{CpG}$ dinucleotide sequences in genomic DNA (Moarefi and Chedin, 2011), and it is divided into three families that are encoded by respective genes, including the DNMT1, DNMT3a, and DNMT3b genes (Kinney and Pradhan, 2011). Different DNMTs play distinct roles in the DNA methylation process. Maintenance of the methylation pattern is achieved by DNMT1 function during DNA replication, whereas de novo methylation is primarily catalyzed by DNMT3a and DNMT3b (Pazienza et al., 2012). DNMT1 is a central component of the epigenetic network that mediates transcription repression (Saunthararajah et al., 2012). Overexpression of DNMT1 promotes global DNA hypermethylation (Biniszkiewicz et al., 2002). In the current study, the concentration of serum DNMT1 in mice and the mRNA expression of DNMT1 in ECs were detected. We found that serum DNMT1 decreased in the HHcy group compared with other groups. Moreover, DNMT1 concentrations in ECs also showed degradation after Hcy stimulation. Decreased expression of DNMT1 and increased activity of SAM further suggests that Hcy enhanced transmethylation.

DNA methylation influences gene expression, and as a result, the function of the altered protein. Accordingly, global DNA hypomethylation, which may induce chromosomal instability, has been associated with various diseases (Chalitchagorn et al., 2004; Suter et al., 2004). Diverse patterns, including both increased and decreased DNA methylation, are seen across several tissues with aging (Yung and Julius, 2008). Several studies have demonstrated global DNA hypomethylation in atherosclerosis. Hypomethylation of the genome mainly affects the intergenic and intronic regions of DNA, particularly the repetitive sequences and transposable elements. B1 repetitive elements, a representative agent of the mice genome and a good predictor of global methylation, are located in a mouse methylation center and provide a target for $d e$ novo DNA methylation (Yates et al., 1999). Therefore, we further determined the methylation status of B1 repetitive elements in blood vessels to investigate the genome methylation status. We demonstrated a decrease in the methylation of B1 repetitive elements in blood vessels. One possible mechanism for this result is that Hcy might have transferred a methyl to SAM through the methionine cycle, and as a result, SAM transferred the methyl to B1 repetitive elements.

Vascular endothelial cell injury has long been known as a preliminary change in the generation of atherosclerosis. Elevation of Hcy contents increases the permeability of human intestinal microvascular endothelial cells through paracellular and transcellular transport pathways (Munjal et al., 2012). After Hcy stimulation, Ox-LDL in ECs increased significantly, which indicated that Hcy might promote LDL oxidization in vitro. Interestingly, the expression of the Ox-LDL receptor, LOX-1, was also upregulated with respect to both mRNA and protein levels. However, methylation of LOX-1 decreased after Hcy stimulation. These observations demonstrated that LOX-1 DNA hypomethylation is a vital mechanism in Hcy-induced atherosclerosis.

Vitamin B12 is an essential micronutrient required for one-carbon metabolism and branched amino acid catabolism, whereas folates play a critical role in maintaining DNA stability by donating one-carbon moieties (Duthie et al., 2010). It is likely that reduced dietary 
methyl donor levels or elevated Hcy levels might lead to more hypomethylation and overexpression of methylation-sensitive genes. Our results showed that serum tHcy, lipids, SAM, and SAH levels all increased, whereas DNMT expression decreased after vitamin B12 and folate treatment in mice. Moreover, expression of LOX-1 in ECs decreased due to demethylation in the presence of vitamin B12 and folate. According to our findings, and based on the effects of vitamin B12 and folates in the methionine cycle, we concluded that vitamin B12 and folate might alleviate Hcy-induced lipid deposition in the vessel wall. These results also demonstrated that LOX-1 might supply methyl groups to other genes, including B1 repetitive elements.

In conclusion, we confirmed that Hcy induced the dysfunction of global DNA methylation in blood vessels in $\mathrm{ApoE}^{-/-}$mice, and that this process is, at least partially, mediated by LOX-1 gene hypomethylation.

\section{ACKNOWLEDGMENTS}

Research supported in part by grants from the National Natural Science Foundation of China (\#81200118, \#81160044, \#81360053), the Provincial Natural Science Foundation of Ningxia (\#NZ13054, \#NZ12185), and the Ningxia Education Department (\#NGY2012063).

\section{REFERENCES}

Biniszkiewicz D, Gribnau J, Ramsahoye B, GaudetF, etal. (2002). Dnmt1 overexpression causes genomic hypermethylation, loss of imprinting, and embryonic lethality. Mol. Cell. Biol. 22: 2124-2135.

Castro R, Rivera I, Struys EA, Jansen EE, et al. (2003). Increased homocysteine and S-adenosylhomocysteine concentrations and DNA hypomethylation in vascular disease. Clin. Chem. 49: 1292-1296.

Chalitchagorn K, Shuangshoti S, Hourpai N, Kongruttanachok N, et al. (2004). Distinctive pattern of LINE-1 methylation level in normal tissues and the association with carcinogenesis. Oncogene 23: 8841-8846.

Delgado-Cruzata L, Wu HC, Perrin M, Liao Y, et al. (2012). Global DNA methylation levels in white blood cell DNA from sisters discordant for breast cancer from the New York site of the breast cancer family registry. Epigenetics 7 : 868-874.

Du X, Han L, Guo AY and Zhao Z (2012). Features of methylation and gene expression in the promoter-associated CpG islands using human methylome data. Comp. Funct. Genomics 2012: 598987.

Duthie SJ, Grant G, Pirie LP, Watson AJ, et al. (2010). Folate deficiency alters hepatic and colon MGMT and OGG-1 DNA repair protein expression in rats but has no effect on genome-wide DNA methylation. Cancer Prev. Res. 3: 92-100.

King WD, Ho V, Dodds L, Perkins SL, et al. (2012). Relationships among biomarkers of one-carbon metabolism. Mol. Biol. Rep. 39: 7805-7812.

Kinney SR and Pradhan S (2011). Regulation of expression and activity of DNA (cytosine-5) methyltransferases in mammalian cells. Prog. Mol. Biol. Transl. Sci. 101: 311-333.

Kirsch SH, Knapp JP, Geisel J, Herrmann W, et al. (2009). Simultaneous quantification of S-adenosyl methionine and S-adenosyl homocysteine in human plasma by stable-isotope dilution ultra performance liquid chromatography tandem mass spectrometry. J. Chromatogr. B Analyt. Technol. Biomed. Life Sci. 877: 3865-3870.

MacLean KN, Greiner LS, Evans JR, Sood SK, et al. (2012). Cystathionine protects against endoplasmic reticulum stressinduced lipid accumulation, tissue injury, and apoptotic cell death. J. Biol. Chem. 287: 31994-32005.

Moarefi AH and Chedin F (2011). ICF syndrome mutations cause a broad spectrum of biochemical defects in DNMT3Bmediated de novo DNA methylation. J. Mol. Biol. 409: 758-772.

Morawietz H (2010). LOX-1 receptor as a novel target in endothelial dysfunction and atherosclerosis. Dtsch Med. Wochenschr 135: 308-312.

Munjal C, Tyagi N, Lominadze D and Tyagi SC (2012). Matrix metalloproteinase-9 in homocysteine-induced intestinal microvascular endothelial paracellular and transcellular permeability. J. Cell Biochem. 113: 1159-1169.

Pazienza V, Tavano F, Benegiamo G, Vinciguerra M, et al. (2012). Correlations among PPARgamma, DNMT1, and DNMT3B expression levels and pancreatic cancer. PPAR Res. 2012: 461784. 
Rapava EA, Akhvlediani MV and Emukhvari MG (2006). Modern view on biochemistry of atherosclerosis. Georgian Med. News 124-128.

Saunthararajah Y, Triozzi P, Rini B, Singh A, et al. (2012). p53-Independent, normal stem cell sparing epigenetic differentiation therapy for myeloid and other malignancies. Semin. Oncol. 39: 97-108.

Suter CM, Martin DI and Ward RL (2004). Hypomethylation of L1 retrotransposons in colorectal cancer and adjacent normal tissue. Int. J. Colorectal Dis. 19: 95-101.

Tehlivets O (2011). Homocysteine as a risk factor for atherosclerosis: is its conversion to S-adenosyl-L-homocysteine the key to deregulated lipid metabolism? J. Lipids 2011: 702853.

Thampi P, Stewart BW, Joseph L, Melnyk SB, et al. (2008). Dietary homocysteine promotes atherosclerosis in apoEdeficient mice by inducing scavenger receptors expression. Atherosclerosis 197: 620-629.

Turcot V, Tchernof A, Deshaies Y, Perusse L, et al. (2012). LINE-1 methylation in visceral adipose tissue of severely obese individuals is associated with metabolic syndrome status and related phenotypes. Clin. Epigenetics 4: 10.

Wernimont SM, Clark AG, Stover PJ, Wells MT, et al. (2011). Folate network genetic variation, plasma homocysteine, and global genomic methylation content: a genetic association study. BMC Med. Genet. 12: 150.

Yates PA, Burman RW, Mummaneni P, Krussel S, et al. (1999). Tandem B1 elements located in a mouse methylation center provide a target for de novo DNA methylation. J. Biol. Chem. 274: 36357-36361.

Yoshimoto R, Fujita Y, Kakino A, Iwamoto S, et al. (2011). The discovery of LOX-1, its ligands and clinical significance. Cardiovasc. Drugs Ther. 25: 379-391.

Yung RL and Julius A (2008). Epigenetics, aging, and autoimmunity. Autoimmunity 41: 329-335. 